\title{
Carotenoids in plant photoprotection
}

\author{
Erich L. Schrott \\ Botanisches Institut der Universität, Menzinger Straße 67, \\ D-8000 München 19, F.R.G.
}

\begin{abstract}
One of the roles of carotenoids in plants which are proposed is to serve as protecting agents against deleterious effects of light. The major mechanisms whereby carotenoids prevent harmful photooxidations in vitro and their suggested protective function in photosynthetic tissue, i.e. chloroplasts, will be summarized briefly. Fungi are non-photosynthetic plants, therefore lack the antenna function of carotenoids but some of them do contain carotenoids located in membranes as well. Thus, fungi can serve as a useful model system to study the potential function of carotenoids in protecting against photosensitized oxidations. Results obtained using the fungus Fusarium aquaeductuum are presented which support the assumption that carotenoids indeed have the capacity to protect in vivo from the deleterious effects of light.
\end{abstract}

\section{INTRODUCTION}

Although it has been recognized for more than half a century that carotenoids apparently are involved in the photoprotection of plants from the harmful effects of light (Ref. 1-5), our insight into the protective function of carotenoids in intact plants is still very small.

Light plays an important role in the development of plants. Its main function with respect to nutrition of most living beings is to serve as an energy source for photosynthesis. The antenna function of carotenoids in harvesting sunlight is reasonably well understood. Light is also used by plants as a signal for triggering biosynthetic and developmental processes. The possible role of carotenoids in acting as photoreceptors in some of these processes has been discussed (Ref. 6-10).

On the other hand, light also causes harmful photoreactions: light which is beneficial, for example to photosynthesis, may also give rise to photoisomerisation or photobleaching of the pigments involved. In intact cells, however, the pigments are bound or sequestered in such a manner as to lower the risk that they undergo damaing photoreactions. But pigments are not the only targets for damaging action of light in a living system. In vitro studies suggest that carotenoids are present in these systems in order to protect them against the deleterious consequences of light by curbing unwanted photoreactions.

\section{IN VITRO REACTIONS}

Deleterious effects of light are described to be wavelenght dependent. Most of the early reports on cell damage were presenting data on effects of $U V-C$ ( $<280 \mathrm{~nm})$; light, which is not part of the solar spectral radiation reaching the surface of the earth. Damage by UV-C is due, among other things, to the formation of pyrimidine dimers in the DNA molecule.

For the discussion of the data presented below it is important to know that there are several mechanisms - at least in bacteria - which overcome the various types of damage. Among these repair mechanisms are the so-called "excision repair", defined as the enzymatic restoration of the dimer damage in the DNA molecule and, most important in our context because they are light dependent, the "photoreactivation" and the "error prone repair". 
Over the years, evidence has increased for the harmful action also of longer wavelength $U V$ and even visible light. To induce damage using visible light, treatment with high intensities and/or doses is required (see Ref. 11, 13 for review; Ref. 14-17 for more recent references). Damage can be reduced when a suitable sensitizing pigment is present in the system. In 1941, Blum described this sensitisation of living tissue by light, air and a sensitiser as the photodynamic effect. What is considered in general to be the mode of action of the photoprotection mediated by carotenoids will be summarized briefly, since it has been reviewed comprehensively during the last years (Ref. 18-21).

The photochemical reactions are initiated by light which excites a sensitizer molecule (s) to its singlet state. It may undergo an intrasystem crossing to form the triplet excited species, ${ }^{3} \mathrm{~S}$. The $3 \mathrm{~S}$ can now initiate a series of reactions, depending on the presence of $0_{2}$ or other potentially active species in the environment. In redox reactions classified as Type I reactions by Gollnick and Schenk (Ref. 22) radical species can be formed, or in Type II reaction the $3 \mathrm{~S}$ can react directly with $0_{2}$ forming singlet oxygen, $1_{0_{2}}$. Among these products are several which can lead to the harmful consequences of the photodynamic effect. ${ }^{1} 0_{2}$ for example causes damage such as cross-linking of membrane proteins, inhibition of the catalytic activity of membrane-bound enzymes, interruption of membrane transport, cell death, mutagenicity or carcinogenicity (Ref. 11, 13, 19, 23).

In vitro, carotenoids do have the capacity to quench the triplet sensitizer, $3^{\text {the }}$ free radical intermediates and also $1_{0_{2}}$. In the case of chlorophyll, $90 \%$ of ${ }^{3} \mathrm{Chl}$ would be quenched by carotenoids, thus intercepting the subsequent photochemical reactions. Due to their triplet energy level, carotenoids containing seven or more conjugated double bonds could be efficient in such an energy transfer from $3 \mathrm{Chl}$ to carotenoids. Carotenoids containing nine or more conjugated double bonds serve as excellent quenchers of $1_{2}$. Chlorophyll is a potent sensitizer for $1_{0_{2}}$ and in photosynthetic tissue the photoprotection by carotenoids is discussed as the dissipation of excess light energy by quenching $3 \mathrm{Chl}$, thus intercepting the formation of $10_{2}$, or by quenching of $10_{2}$ itself (Ref. 24,25). As an additional function of carotenoids in chloroplasts, their possible role in the structural stabilisation of the photosynthetic membranes was discussed (Ref. 24).

\section{IN VIVO STUDIES}

In order to study the functions of carotenoids in vivo, it seems profitable to reduce as far as possible the number of parameters influenced by the factor light. Among the plant kingdom, fungi do not contain chloroplasts and therefore lack the antenna function of carotenoids, but some of them do contain carotenoids as a membrane component. In the fungus Fusarium aquaeductuum, the formation of carotenoids is light dependent and occurs within a few hours after a short light pulse of the order of minutes has been given (Ref. 10, 26). Thus, from genetically and physiologically the same cell population, aliquots of mycelia containing and not containing carotenoids are available, avoiding disadvantages of mutant studies.

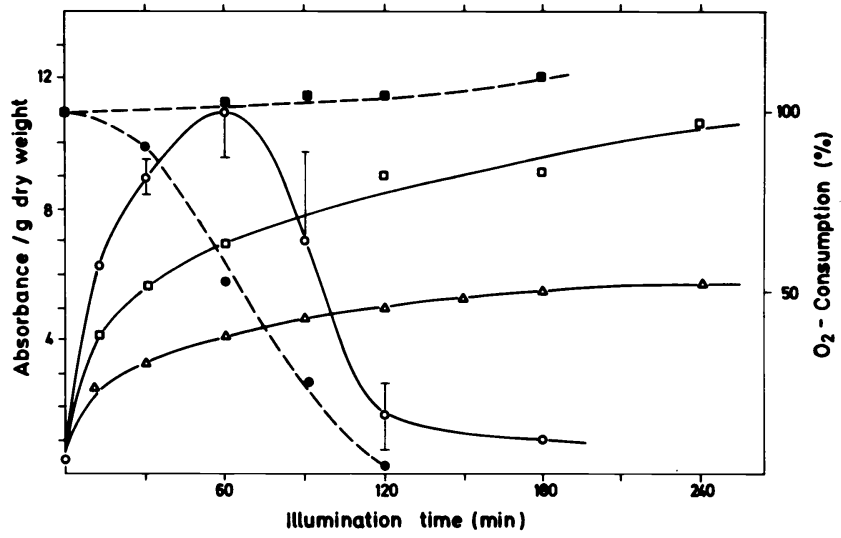

Fig. 1. Amount of carotenoids induced in Fusarium mycelia by different light sources and fluence rates: $\square-\square$ white light $\left(2 \times \frac{20 \mathrm{Wm}^{-2}}{2}\right) ; \Delta-\Delta U V-A\left(1 \mathrm{Wm}^{-2}\right) ; 0-0$ UV-A ( 2 x $\left.28 \mathrm{Wm}^{-2}\right)$. White or UV-A light was obtained from two banks of 0 sram $L 19$ fluorescent tubes or 0 sram $L$ 73, respectively, from each side of the sample (Ref. 27). Oxygen consumption of the mycelium at the end of the particular illumination period: - UV-A $(2 \times 28 \mathrm{Wm}-2) ; \square-0$ dark control. 
As a prerequisite for studies on the protective role of carotenoids in Fusarium some harmful effects of light were characterized. When white light or low fluence rates of UV-A $\left(2 \times 0.4 \mathrm{Wm}^{-2}\right)$ were administered the more carotenoids were formed during the subsequent $24 \mathrm{~h}$ dark incubation time the longer the irradiation occurred (Fig. 1; Ref. 26, 28). Using relatively high fluence rates of UV-A (2 $\left.x 28 \mathrm{Wm}^{-2}\right)$ the formation of enhanced amounts of carotenoids was induced upon illumination time periods up to $60 \mathrm{~min}$. However, further illumination apparently gave rise to photodamaging events indicated by the decreasing capability for subsequent carotenoid formation resulting in an optimum curve. The position of the optimum on the time scale strongly depends on the physiological state of the organism. Although greatest care was taken for standardization of the growth conditions, the physical age does not coincide exactly with the physiological age in every experiment or group of experiments. This explains minor discrepancies in the time periods required for optimum carotenoid production apparent in Fig. 1, 4 and 5. Fig. 1 also shows that oxygen consumption of the mycelia apparently decreases immediately after treatment with UV-A light.

In order to test for harmful effects of light on basic biosynthesic cell processes, we examined the time course of uptake and incorporation of radioactively labelled precursors into protein or nucleic acids. As an example the inhibition of leucine transport and metabolism by white light is depicted in Fig. 2: treatment of Fusarium mycelia with cell destructive and protein precipitating trichloroacetic acid (TCA) solution after different times of incubation with $\left({ }^{14} \mathrm{C}\right)$-leucine before and after the onset of light reveals that leucine incorporation is reduced in white light emmitted by the fluorescent tubes. When bright midday sunlight is applied incorporation of leucine comes to a complete stop. This is in agreement with the observation that exposure to sunlight for $30-60 \mathrm{~min}$ is sufficient to cause cell death. On the other hand, when mycelia are washed with distilled water omitting TCA the cells are left intact and the total amount of (14C)-leucine "trapped" within the hyphae can be measured. By use of this procedure it was found that leucine uptake completely ceases in white light $\left(2 \times 20 \mathrm{Wm}^{-2}\right)$ for about $2 \mathrm{~h}$. 'lhis inhibitory effect decreases with longer illumination time indicating a restoration of the uptake mechanism.

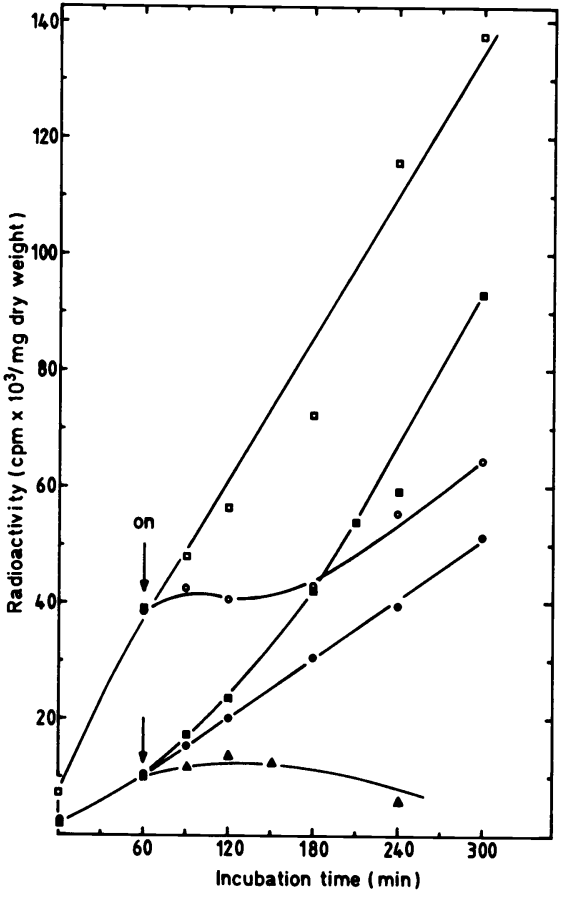

Fig. 2 Uptake and incorporation of $\left({ }^{14} \mathrm{C}\right)$-leucine before and after the treatment with white light. Trapped leucine (see text): $0-02 \times 20 \mathrm{Wm}^{-2}$; $\square-\square$ dark control. Incorporated leucine: - $-2 \times 20 \mathrm{Wm}^{-2} ; \Delta-\Delta$ midday sunlight; $\square$ dark control.

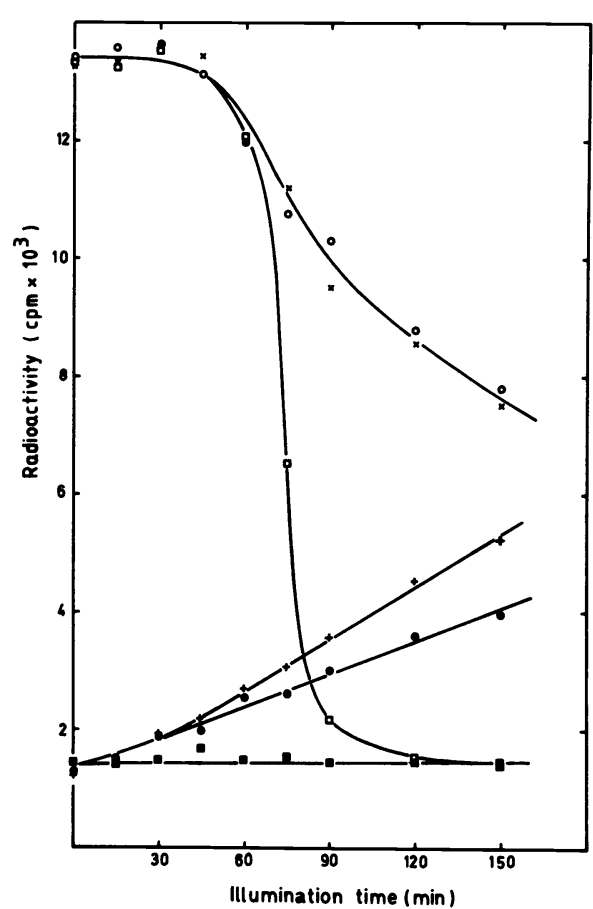

Fig. 3. Time course of incorporation and release of trapped (14C)-leucine as a function of the treatment during incubation. Incorporated leucine: +-+ dark control; $0-0$ white light $\left(2 \times 20 \mathrm{Wm}^{-2}\right) ; \square-\square$ UV-A $\left(2 \times 28 \mathrm{Wm}^{-2}\right)$. Trapped leucine: $\mathrm{X}-\mathrm{X}$ dark control; $-\bullet$ white light

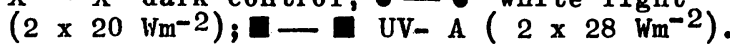


More direct evidence for membrane damage as a result of irradiation is derived from pulse experiments. After removal of the leucine from the medium the time course of metabolism of the trapped leucine was followed. Fig. 3 shows that the incorporation of pool leucine (i.e., non-incorporated leucine within the cell) into protein is reduced upon illumination in agreement with the results in Fig. 2. Using UV-A light ( $2 \times 28 \mathrm{Wm}^{-2}$ ) the incorporation is completely inhibited. The amount of trapped leucine is unaffected by white light whereas at the same time UV-A light causes a dramatic decrease in the amount of trapped leucine down to the level of the amount of leucine already incorporated. This efflux reflects the breakdown of the diffusion barrier, since it also occurs under nitrogen atmosphere rendering an active efflux unlikely (the ability of the mycelia to perform a nitrogen sensitive active efflux was shown in experiments beyond the scope of this article).

Now by means of the tools described above the role of carotenoids in preventing damaing effects of 1 ight was investigated.

Mycelia of Fusarium were illuminated with white light for 0,3 and 100 min giving rise to different amounts of carotenoids at the end of a $4 \mathrm{~h}$ incubation period. Subsequently the samples were illuminated using damaging UV-A light (see Fig. 1). Fig. 4 clearly demonstrates that the capability of mycelia to synthesize carotenoids during the following $24 \mathrm{~h}$ dark incubation period is preserved for longer UV-A irradiation time the longer the pre-illumination was performed. Correspondingly the effectiveness of UV-A light in inhibiting the respiration decreases. It is worth noting that similar results were obtained when UV-B light ( $2 \times 30 \mathrm{Wm}^{-2}$; filtered Philips TL 12) was administered, which produces essentially the same damaging characteristics as UV-A (data not shown).

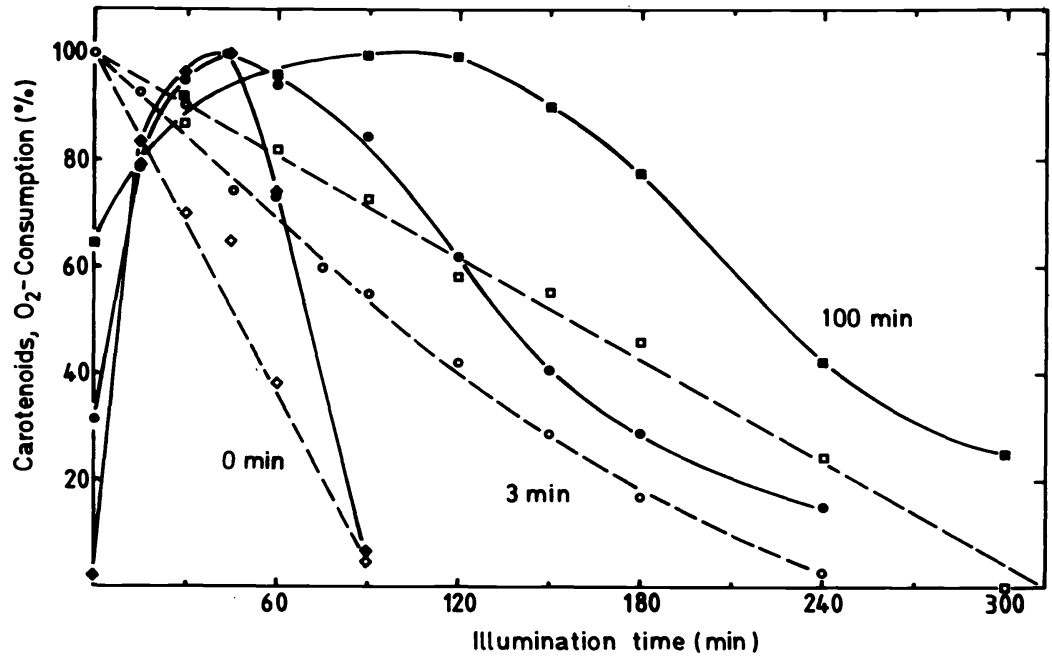

Fig. 4. Amounts of carotenoids induced by UV-A $\left(2 \times 28 \mathrm{Wm}^{-2}\right)$ irradiation 4 h after pre-illumination with white light for the time periods indicated (continuous lines), measured $24 \mathrm{~h}$ after induction. Oxygen consumption of these mycelia at the end of the particular UV-A irradiation period (dashed lines).

Inhibition of uptake and incorporation of an amino acid $\left(\left({ }^{35} \mathrm{~s}\right)\right.$-methionine compatibly substituted for $\left({ }^{14} \mathrm{C}\right)-\mathrm{leucine}$ in the later experiments) is also distinctively diminished when mycelia were pre-illuminated (Fig. 5; data of UV-B treatment shown). Moreover, following the time course for longer periods of time it becomes evident that at least the amount of trapped methionine increases again when the mycelium was pre-illuminated, suggesting a restoration process is taking place. 
Fig. 5. Uptake and incorporation of (35s)-methionine into pre-illuminated mycelia (procedure as in Fig. 4) as a function of UV-B ( $\left.2 \times 30 \mathrm{Wm}^{-2}\right)$ irradiation time and subsequent dark incubation.

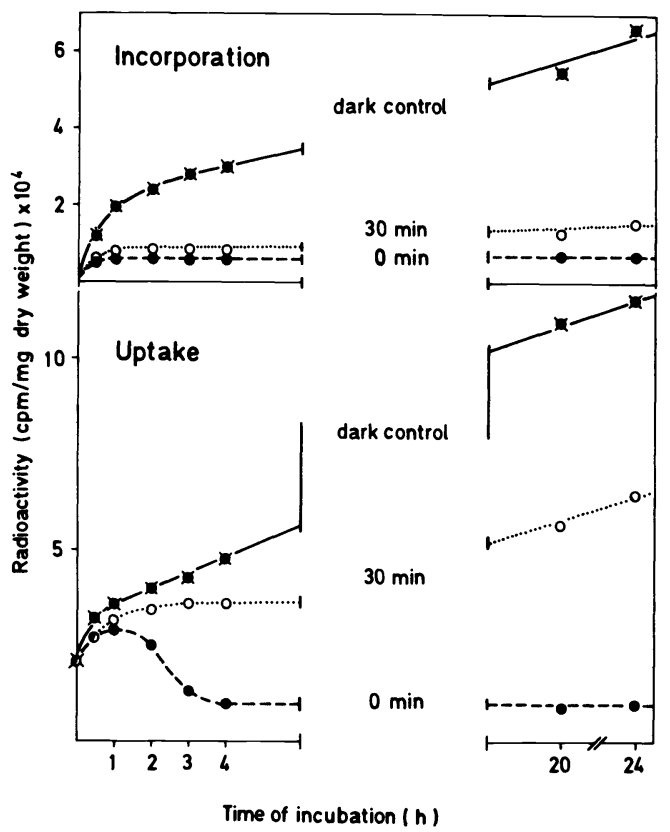

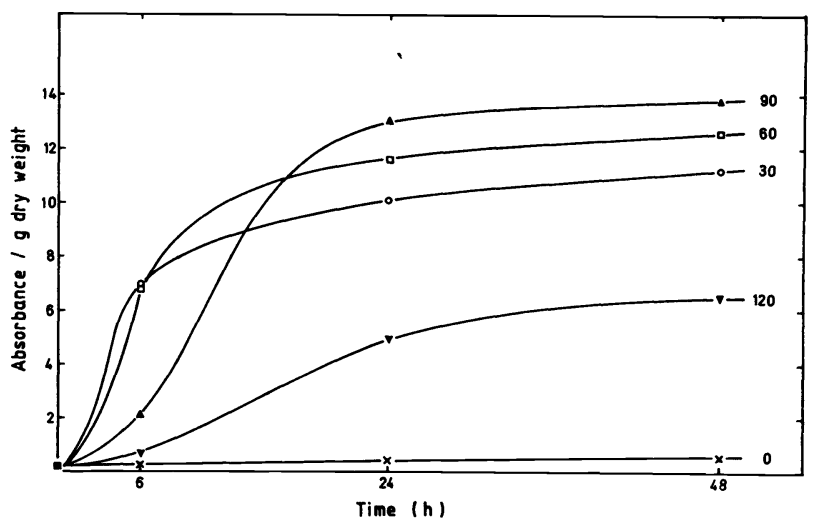

Fig. 6. Time course of accumulation of carotenoids induced by different UV-A (2.x $\left.28 \mathrm{Wm}^{-2}\right)$ irradiation periods. $0-030$ min; $\square-\square 60 \min ; \Delta-\Delta 90 \min$; $\nabla-\nabla 120$ min; $\mathrm{X}-\mathrm{X}$ dark control.

Restoration was already found in the experiments illustrated in Fig. 2 and is also demonstrated by the following results. Investigation of the time course of carotenoid formation after treatment with UV-A light revealed that the accumulation kinetics are different for the illumination times applied (Fig. 6). After $24 \mathrm{~h}$ of incubation the maximum amounts of carotenoids were synthesized when light was given for $90 \mathrm{~min}$. The rate of synthesis, however, is remarkably reduced during the initial phase when compared with the shorter illumination periods, which attests that the mycelia first hampered in carotenoid production are able to recover and ultimately form the appropriate amounts of carotenoids.

By the results shown in Fig. 4 and 5 a protective role of carotenoids seems to be well documented. However, it cannot be ruled out as yet that the apparent damage delays upon pre-illumination are not, or are not only, due to carotenoid protection but are caused by a repair mechanism which is light inducible. Carotenogenesis in Fusarium is brought about by wavelengths $<520 \mathrm{~nm}$ (Ref. 26). Reducing the spectral range of the white light used to the portion $<520 \mathrm{~nm}$ leaves the damage delays unchanged. Therefore the probability for a photoinducible repair being involved is reduced to a blue/UV inducible species. Nevertheless it still implies that blue/UV irradiation might be able to establish a protection either by photoinduction of carotenoid biosynthesis or by photoinduction of a repair system or by both of them. 
To overcome these difficulties two experimental approaches avoiding light as the trigger for carotenoid production are envisaged: (1) applying p-chloro-mercuribenzoate, which substitutes for light in inducing carotenoid synthesis in Fusarium (Ref. 29) and (2) turning back to mutant studies despite their disadvantages in terms of physiological compatibility and making use of Fusarium mutants which lack photocontrol and therefore synthesize large amounts of carotenoids in the dark (Ref. 30). We are confident that these experiments will clarify the role of carotenoids in photoprotection.

\section{REFERENCES}

1. K. Noak, Z. Bot. 17, 481 (1925).

2. K. Noak, Naturwissenschaften 14,383 (1926).

3. II. Claes, Z. Naturforsch. 2 b, 461 (1954).

4. 0. Kandler and F. Schötz, Z. Naturforsch. 11b, 708 (1956).

5. D.S. Robertson, M.D. Bachmann and I.C. Anderson, Photochem. Photobiol. 5, 795 (1966).

6. W. Shropshire, jr., in H. Senger, Ed., The Blue Light Syndrome, p. 172, Springer, Berlin (1980).

7. E. De Fabo, in II. Senger, Ed., The Blue Light Syndrome, p. 187, Springer, Berlin (1980).

8. J. Gressel and W. Rau, in W. Shropshire, jr., and H. Mohr, Eds., Encycl. Plant Physiol., Vol. 16, p. 603. Springer, Berlin (1983).

9. P.-S. Song, in H. Senger, Ed., Blue Light Effects in Biological Systems, p. 75, Springer, Berlin (1984).

10. W. Rau, this volume.

11. R.B. Webb, in K.C. Smith, Ed., Photochemical and Photobiological Reviews, Vol. 2, p. 169. Plenum Press, New York (1977).

12. W.M. Generoso, M.D. Shelby and F.J. de Serres, DNA Repair and Mutagenesis, Plenum Press, New York (1980).

13. J. Jagger, Photochem. Photobiol. 34, 761 (1981).

14. J.G. Peak, C.S. Foote and M.J. Peak, Photochem. Photobiol. 24, 45 (1981).

15. F. Zölzer and J. Kiefer, Photochem. Photobiol. 37, 39 (1983).

16. M. Holmberg, Photochem. Photobiol. 27, 293 (1983).

17. L.R. Kelland, S.H. Moss and D.J.G. Davies, Photochem. Photobiol. 37, 301 (1983).

18. N.I. Krinsky, Pure \& Appl. Chem. 51, 623 (1979).

19. N.I. Krinsky, in H.H. Wasserman and R.W. Murray, Eds., Singlet Oxygen, p. 597. Academic Press, New York (1979).

20. N.I. Krinsky, in II.-0. Kim and P.-S. Song, Eds., Topics in Photobiology, Jeju National University, Jeju (1983).

21. N.I. Krinsky, in W. Bors, M. Saran and D. Tait, Eds., Oxygen Radicals in Chemistry and Biology, p. 453. Walter de Gruyter \& Co., Berlin (1984).

22. K. Gollnik and G.0. Schenk, in J. Hamer, Ed., 1,4-Cycloaddition Reactions, p. 255. Academic Press, New York (1967).

23. C.S. Foote, in A.P. Autor, Ed., Pathology of Oxygen, p. 21. Academic Press, New York (1982).

24. P. Mathis and C.C. Schenk, in G. Britton and T.W. Goodwin, Eds., Carotenoid Chemistry and Biochemistry, p. 339. Pergamon Press, New York (1979).

25. S.M. Ridley, in G. Britton and T.W. Goodwin, Eds., Carotenoid Chemistry and Biochemistry, p. 353. Pergamon Press, New York (1979).

26. W. Rau, Planta 72, 14 (1967).

27. E.L. Schrott, planta 150, 174 (1980).

28. A. Huber and E.L. Schrott, in H. Senger, Ed., The Blue Light Syndrome, p. 299. Springer, Berlin (1980).

29. W. Rau, P1anta 74, 263 (1967).

30. W. Rau, B. Feuser and A. Rau-Hund, Biochim. Biophys. Acta 136, 589 (1967). 